\title{
Predictors of Quality of Life in Patients with Heart Disease
}

\author{
Mohammad Ali Soleimani ${ }^{1}$ (D) Simin Zarabadi-Pour ${ }^{1}$ (D) \\ Seyedeh Ameneh Motalebi ${ }^{1}$ (D) Kelly-Ann Allen ${ }^{2}$ (D)
}

(c) Springer Science+Business Media, LLC, part of Springer Nature 2020

\begin{abstract}
Individuals with heart disease have been found to have more negative psychological and physical effects that impact their quality of life (QoL) than the general population. Spiritual well-being is considered a protective factor associated with QoL in people with heart disease. Therefore, the current research seeks to evaluate whether sociodemographic factors and spiritual well-being predict QoL among patients with heart disease. A total of 500 patients who were selected through a convenient sampling method from an Iranian hospital participated in this descriptive-correlational study. Data were collected using the McGill QoL Questionnaire, the Spiritual Wellbeing Scale, and demographic variables. The data analysis included descriptive and inferential statistics powered by SPSS (v. 23). Following multivariate analyses, findings revealed that those participants with their main source of income derived from family or a government pension and with College or intermediate educational levels were more likely to have higher QoL. Those participants with average or poor socioeconomic status reported higher QoL than those who were more affluent. Furthermore, younger patients $(-0.2,95 \% \mathrm{CI}-0.3$ to $-0.003, p=0.016)$ and those with higher social support $(0.7,95 \% \mathrm{CI} 0.2$ to $1.3, p=0.006)$ and spiritual well-being $(0.2,95 \%$ CI 0.1 to $0.3, p<0.001)$ had significantly better QoL. In the current study, spiritual well-being and social support led to reduced negative psychological sequelae and improved QoL in cardiac patients.
\end{abstract}

Keywords Quality of life - Spirituality - Social support · Heart disease

\section{Affiliations}

\section{Mohammad Ali Soleimani ${ }^{1}(\mathbb{D})$ Simin Zarabadi-Pour ${ }^{1}$ (D) . Seyedeh Ameneh Motalebi ${ }^{1}$ (D) Kelly-Ann Allen ${ }^{2} \mathbb{D}$}

Mohammad Ali Soleimani

ma.soleimany@qums.ac.ir

Simin Zarabadi-Pour

s.zarabadipour@qums.ac.ir

Kelly-Ann Allen

Kelly-ann.allen@monash.edu

1 Social Determinants of Health Research Center, Research Institute for Prevention of Non-Communicable Diseases, Qazvin University of Medical Sciences, Qazvin, Iran

2 Educational Psychology and Inclusive Education, Faculty of Education, Monash University and The Centre for Positive Psychology, The Melbourne Graduate School of Education, The University of Melbourne, Parkville, Australia 